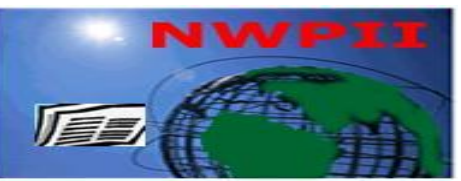

American Journal of Biomedical Sciences

ISSN: 1937-9080

nwpii.com/ajbms

\title{
Control of Prosthetic Device Using Support Vector Machine Signal Classification Technique
}

\begin{abstract}
J.M. Fontana and A.W.L. Chiu*
College of Engineering and Science, Biomedical Engineering Department, Louisiana Tech University, Ruston, LA, USA

*Corresponding author:

Dr. Alan Chiu.

College of Engineering and Science, Biomedical Engineering Department, Louisiana Tech University

Ruston, LA, USA.

Tel: (318) 257-5231.

E-mail: alanchiu@latech.edu

Received: 26 March 2009; | Revised: 29 May 2009; | Accepted: 25 June 2009

Abstract

An appropriate classification of the surface myoelectric signals (MES) allows people with disabilities to control assistive prosthetic devices. The performance of these pattern recognition methods significantly affects the accuracy and smoothness of the target movements. We designed an intelligent Support Vector Machine (SVM) classifier to incorporate potential variations in electrode placement, thus achieving high accuracy for predictive control. MES from seven locations of the forearm were recorded over six different sessions. Despite meticulous attempt to keep the recording locations consistent between trials, slight shifts may still occur affecting the classification performance. We hypothesize that the machine learning algorithm is able to compensate for these variations. The recorded data was first processed using Discrete Wavelet Transform over 9 frequency bands. As a result, a 63-dimension embedding of the wavelet coefficients were used as the training data for the SVM classifiers. For each session of recordings, a new classifier was trained using only the data sets from the previous sessions. The new classifier was then tested with the data obtained in the current session. The performance of the classifier was evaluated by calculating the sensitivity and specificity. The result indicated that after a critical number of recording sessions, the classifier accuracy starts to reach a plateau, meaning that inclusions of new training data will not significant improve the performance of the classifier. It was observed that the effect of electrode placement variations was reduced and that the classification accuracy of $>89 \%$ can be obtained.
\end{abstract}

Keywords: Myoelectric control; Electrode placement; Support Vector Machine; Multi-state classification. 


\section{INTRODUCTION}

Myoelectric signals (MES) recorded on the body surface presents information related to the neuromuscular activity from which it originates. These signals can then be used to determine when the contraction of a muscle or if an intention of a movement exists. This is an important tool because it allows the control of assistive devices by means of voluntary contractions produced by the subject. People with disabilities (i.e. amputee, cerebral palsy, spinal cord injury, etc) will then have the opportunity of not only communicating with the external environment but also the ability to perform tasks that would otherwise be impossible.

Since the 1960s, researchers had been trying to address different alternatives for discriminating different muscle states that may lead to an improvement in the acceptance and performance of myoelectric prostheses. These studies were mainly focused on three major factors that affect the acceptance rate by the users: the type of prosthesis, the degree of training needed by the user and the type of myoelectric control used [1].

Regarding the control approach, conventional myoelectric control systems extract the information of the muscle activity either from its intensity or from its rate of change. These features are used to initiate a set of commands that can be used to instruct the prosthetic device for a specific degree of freedom $(\mathrm{DoF})$ in movement. The main drawbacks of this control strategy are that it requires source signal independence and that only a reduced number of functions of the prosthesis can be controlled [2]. To overcome these limitations, pattern recognition based myoelectric control systems attempt to discriminate different classes of movements, where each class is directly related with a DoF in movement. Thus, the user can voluntarily select and modulate the functions of the prosthetic device.

The pattern recognition process encompasses a series of steps before the final control outcome can be achieved. They are represented for each one of the blocks illustrated in Figure 1.

The two most important factors affecting the final performance of the pattern recognition-based control system are the feature extraction and signal classifier [1]. In order to obtain an acceptable accuracy at the end of the process, the classifier has to be appropriately trained. It has been demonstrated that the accuracy of the pattern recognition system would be compromised when possible shifts in the electrode location are not taken into account [3]. In addition, TimeFrequency representations (TFR) of the electromyographic (EMG) signal present better performance than time domain representations (TD) when both techniques are used to extract information from that signals [4].

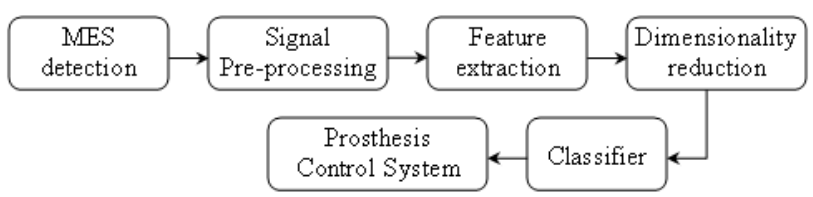

Fig. 1 Block diagram of the pattern recognition process for the control of a prosthetic device is given. The surface EMG signal is detected and conditioned for the feature extraction. A classifier takes the reduced set of features and determines the appropriate action of the prosthesis. The control system interprets the output of classifier and controls the mechanical components of the prosthetic device.

Consequently, the objective of this work is to design an intelligent classifier that incorporates in the training process both variations on the electrode placement and the TFR information from the MES. The proposed myoelectric signal classification system is an implementation of a support vector machine (SVM) classifier that is able to discriminate between four different wrist movements and one rest position. It is trained on the feature space consisting of the averaged wavelet coefficients extracted from seven MES channels distributed along the five forearm muscles. The performance of the new classifier is evaluated by calculating the accuracy, that is, the number of test points that are correctly classified over the total number of test points.

To assimilate the potential variations of electrode placements a new training strategy is implemented. In the case of a prosthetic device, these variations may be the result of a misalignment between the socket and the residual limb or a slight change in the electrode position when the prosthesis is used in various orientations 
[3]. To simulate those deviations, EMG signals were recorded during consecutive experimental sessions. From one session to another, slight shifts (no more than $3 \mathrm{~cm}$ ) in the electrode location are expected when they had been removed and replaced. Following each recording, a classifier is trained and tested with the information from the previous and current sessions, respectively. After a critical number of recordings, the pattern recognition system was able to adapt to the shifts in the electrode placement, meaning that the classification accuracy will not be significantly affected by the addition of new training data. As a result, the prosthetic device can be commanded accurately despite of possible electrode placement variations.

\section{Materials AND MethodS}

The block diagram presented in Fig. 1 will be taken as a reference for introducing the materials and methods used.

\section{A. MES signal detection and Signal Pre-processing}

Myoelectric signals from seven locations of the forearm were recorded over six different sessions on six healthy subjects. One pair of $\mathrm{Ag} / \mathrm{AgCl}$ electrodes (Vermed, Inc) were placed on the Extensor Digitorum, Anconeous, and Flexor Carpi Radialis muscles and two pairs of electrodes were placed on the Extensor Carpi Ulnaris and the Flexor Carpi Radialis muscles. Each bipolar configuration had $2 \mathrm{~cm}$ of inter-electrode distance and was aligned with the muscle fibers in order to record valuable information near a specific muscle on the forearm, as shown in Figure 2. The reference electrode was placed on the wrist.

On each recording session, the subjects were asked to perform four different wrist movements: Abduction, Adduction, Extension and Flexion (illustrated in Figure 3). For each movement, five repetitions of 5 seconds duration each had to be completed with 3 seconds of resting intervals between each repetition to avoid muscle fatigue. The same procedure was repeated at least five times. As a result, four movements of 125 seconds of signals corresponding to isometric contractions were collected from seven channels and then stored. Finally, 60 seconds of signals were collected with the forearm upholding and 60 seconds with the forearm resting Am. J. Biomed. Sci. 2009, 1(4), 336-343; doi: 10.5099/aj090400336 besides the body. These recordings constituted the Rest class. All the data underwent an anti-aliasing low pass filter before being acquired wirelessly at a sample rate of $960 \mathrm{~Hz}$ and at 12-bit resolution.

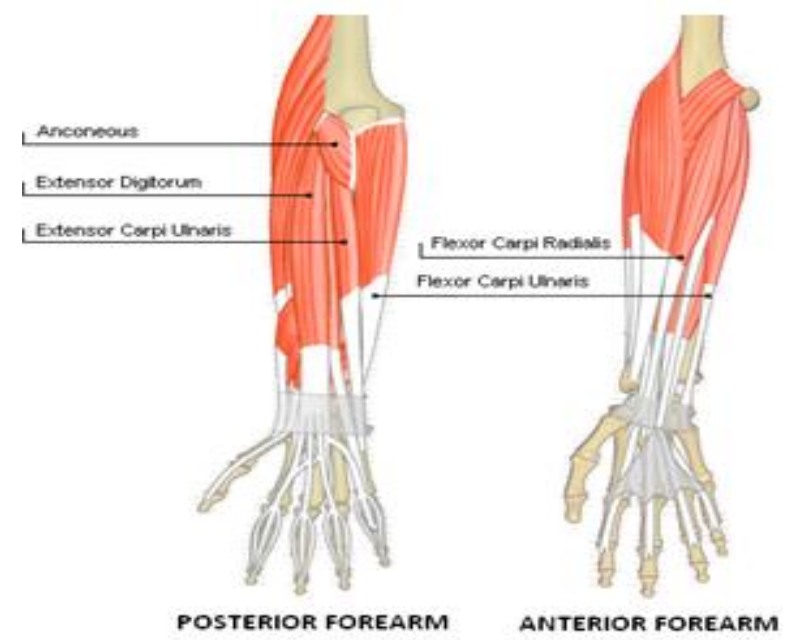

Fig. 2 EMG signals were collected using seven bipolar channels distributed over five forearm muscles when performing wrist movements.

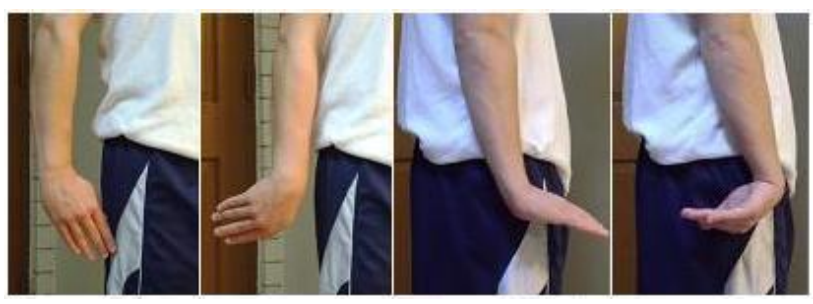

Fig. 3 Four specific wrist movements representing four of the classes to be discriminated: Abduction, Adduction, Extension and Flexion respectively.

Using MATLAB 7.3.0 (R2006b) software (The MathWorks Company), the signals were then conditioned for extracting the features. All repetitions were filtered with a tenth order highpass Butterworth filter ( $10 \mathrm{~Hz}$ cutoff frequency) in order to eliminate low frequency motion artifacts. A notch filter $(60 \mathrm{~Hz})$ was also applied. The amplitude of the recorded signal is normalized in non-overlapping 256-point windows.

\section{B. Feature Extraction and Dimensionality Reduction}

Due to the non-stationary characteristics of the collected signals, Discrete Wavelet Transform (DWT) was applied to each 256-point moving time window segment (corresponding to $266 \mathrm{msec}$ ) of muscle contraction.

(C) 2009 by NWPII. All rights reserved. 
Given the discrete vector $y[n] ;(n=1,2, \ldots$, $N)$, representing the EMG signal in each time window segment, the DWT maps this vector to a set of wavelets coefficients, whose number depends on the level of depth decomposed. In this case, as the windowed signal contain $N=256$ points, a full decomposition will allow a set of $j=\log _{2} N=8$ levels of detailed coefficients and one level of approximation coefficients. Therefore, information from 9 frequency bands was obtained when performing full wavelet decomposition using Coiflet mother wavelet of the second order during the preprocessing stage.

The success of any pattern recognition process depends on the set of features presented to the classifier. A small training set given to the classifier led to a classifier with better generalization [4] but possibly sub-optimal performance. Too much information in the training set will lead to overfitting in the classification. Therefore, it is important to reduce the dimension of the information presented to the classifying during the wavelet decomposition. In order to accomplish that, the energy of the wavelet coefficients was averaged at each resolution level. The resulting values for each recording channel were combined into a single vector to give a 63dimension ( 9 frequency bands over 7 channels) embedding of the wavelet coefficients.

\section{SVM classifier}

The Support Vector Machines (SVM) is a machine learning algorithm that maps the input data into a high dimension feature space, in which the different dynamics are linearly separable. Then, a linear decision surface (hyperplane) is created in the new feature space [5]. This new model can be represented by the following equation:

$$
y(\mathbf{x})=\mathbf{w}^{T} \phi(\mathbf{x})+b
$$

where $\mathbf{w}$ and $b$ are the parameters of the hyperplane and $\phi(\mathbf{x})$ represents the feature space transformation.

Considering a two-class classification problem, a data point $\mathbf{x}_{n}$ may have two possible targets: $t_{n}= \pm 1$. Assuming that the training data is linearly separable in the feature space, the hyperplane created will exactly separate them. Therefore, Eq. (1) will satisfy $y\left(\mathbf{x}_{n}\right)>0$ for points with $t_{n}=+1$ and $y\left(\mathbf{x}_{n}\right)<0$ for points with $t_{n}=-1$. Moreover, all points will satisfy the condition:

$t_{n} \cdot y\left(\mathbf{x}_{n}\right)>0$

Several solutions may be found to separate the classes exactly. The SVM theory attempts to find the solution that gives the smallest generalization error, which means that the model created will be able to categorize correctly a wide variety of new input data [6].

The operation of the SVM is based on the concept of margin. It is defined as the smallest distance between the separating hyperplane (decision boundary) and any of the points. That distance is given by:

$\frac{t_{n} \cdot y\left(\mathbf{x}_{n}\right)}{\|\mathbf{w}\|}=\frac{t_{n} \cdot\left(\mathbf{w}^{T} \phi\left(\mathbf{x}_{n}\right)+b\right)}{\|\mathbf{w}\|}$

The objective of this approach is to find the optimal parameters $\mathbf{w}$ and $b$ that maximizes this distance, therefore maximizing the margin. It requires the solution of the following quadratic optimization problem:

$$
\min _{\mathbf{w}, b}\left\{\frac{1}{2}\|\mathbf{w}\|^{2}\right\}
$$

which is subject to the following constrains satisfied for all of the training points:

$t_{n} \cdot\left(\mathbf{w}^{T} \phi\left(\mathbf{x}_{n}\right)+b\right) \geq 1 \quad n=1, \ldots, N$

with $N$ being the total number of training points. For the point that closest to the surface:

$t_{n} \cdot\left(\mathbf{w}^{T} \phi\left(\mathbf{x}_{n}\right)+b\right)=1$

The solution is obtained by calculating the Lagrange multipliers $a_{n}$, with one multiplier for each constraint in Eq. (5). Thus, each data point is associated with a multiplier. Training points for which $a_{n}=0$ are not taken into account for the final model. The remaining points satisfy Eq. (6) and are used for predicting outputs for new input data. These points define the decision boundary and are called support vectors. They are located on the maximum margin hyperplane in feature space.

Conversely, the solution can not be reached if the training data is not linearly 
separable in the feature space $\phi(\mathbf{x})$. Thus to solve the new problem, the constraints in Eq. (5) have to be relaxed for allowing some training points to be on the wrong side of the decision boundary (soft margin). It is achieved by introducing a penalty term $\xi_{n} \geq 0$ for each training point. It is called slack variable and its value increase with the distance from the margin boundary. Thus, $\xi_{n}=0$ for points on or inside the correct margin; $\xi_{n}<1$ for points that lie between the decision boundary and the correct margin and $\xi_{n}>1$ for point on the wrong side of the decision boundary (misclassified points)

Now the optimization problem becomes:

$$
\min _{\mathbf{w}, b}\left\{C \cdot \sum_{m=1}^{M} \xi_{m}+\frac{1}{2}\|\mathbf{w}\|^{2}\right\}
$$

subjected to the constraints:

$$
\begin{aligned}
& t_{n} \cdot\left(\mathbf{w}^{T} \phi\left(\mathbf{x}_{n}\right)+b\right) \geq 1-\xi_{n} \quad n=1, \ldots, N \\
& \xi_{n} \geq 0
\end{aligned}
$$

where $C>0$ is the parameter that controls the trade off between the misclassification error and the complexity of the model.

SVM were originally designed to discriminate between two classes. However, several methods have been introduced to solve multiclass classification problems. Some of them consider all classes at once and some combine various binary classifiers to create the multiclass classifier. Between them, it has been shown that "oneagainst-one" (1-v-1) and "directed acyclic graph" (DAG) [7] methods are more appropriate for practical use [8]. The DAG method overcomes the limitation of the "1-v-1" strategy in terms of the evaluation time needed. It requires less computation time to perform the testing stage, which is an important point for the implementation of the pattern recognition system in real time. Another advantage of the DAG approach is that for a multiclass classification problem, the bound on the generalization error depends on the number of classes $(\mathrm{K})$ and not on the dimension of the space.

For this study, the DAG method was implemented to classify $K=5$ movements. In the training stage, one binary classifier is constructed for each pair of movements, leading to a total of $K(K-1) / 2$ binary classifiers. When training each binary classifier, the data corresponding to the two different classes may overlap, therefore, the class separation has to be performed using the soft margin approach. Thus, the training stage will involve the solution of the quadratic optimization problem presented in Eq. (7) with inequality constrains. Several algorithms have been designed for solving this type of problem. Among them, sequential minimal optimization (SMO) is one of the most popular approaches addressed to train support vector machines. It was used in this pattern recognition system to train 10 different binary classifiers. Both the kernel function used to map the input data (Radial Basis Function was chosen) as well as the value of the constant $C$ were the parameters required for this algorithm.

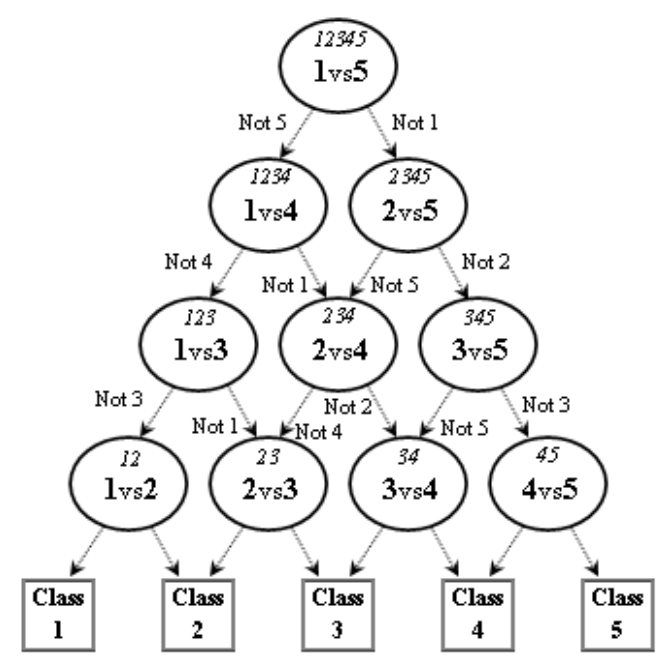

Fig. 4 The decision directed acyclic graph used to discriminate between five classes.

In the testing phase, a rooted binary directed acyclic graph is used to determine the movement that originated the input data. As illustrated in Figure 4, our classifier contains 5 number of leaves and $K(K-1) / 2$ number of internal nodes. The decision tree that the DAG created in this study can classify four wrist movements and the resting position. Inside each decision node, the list state is placed at the top, and the two classes being evaluated are shown at the center. Each node is associated with a binary classifier. As it is stated in [7], this method is similar to operating it 
on a list. It starts with all classes at the root node, where the new testing point $\mathbf{x}$ is first evaluated against the first and the last classes on the list. The class that is not preferred by the node is eliminated from the list and a new list is created for the next node. Therefore, the test point will go through an evaluation path containing $(K-1)$ internal nodes before reaching the leaf that contains only one final class on the list.

In order to incorporate the information corresponding to the electrode placements variations, one set of ten binary classifiers is trained on each recording session. The first set for every subject is trained on the second session using all the data recorded on session one. After training, all the data recorded in the current session is used for testing using the decision DAG method. The same rule is applied for subsequent sessions, the training and testing sets are created with data from previous and current sessions respectively.

\section{Results}

The performance of the classifier sets was evaluated by calculating the numbers of points of the testing set that were correctly classified. This is referred to as the classifier accuracy. Figure 5 illustrates the accuracy of the multi-state classifier after each training session. The values were averaged over the six subjects. Because the classifier of a specific session is trained using data from previous ones, the first point of this figure corresponds to session 2 . The results indicate that a minimum of three recording sessions are required to achieve accuracy higher than $89 \%$. After training the multiclass classifier with data from two sessions (recording session \#3) the classifier accuracy starts to reach a plateau, so it does not increase considerably after that point.

Figure 6 illustrates the improvement in accuracy for the classification of each movement. Except for the Flexion movement, the accuracies of the remaining classes (Abduction, Adduction, Extension and Rest) are higher than $85 \%$ after only three sessions of recordings.

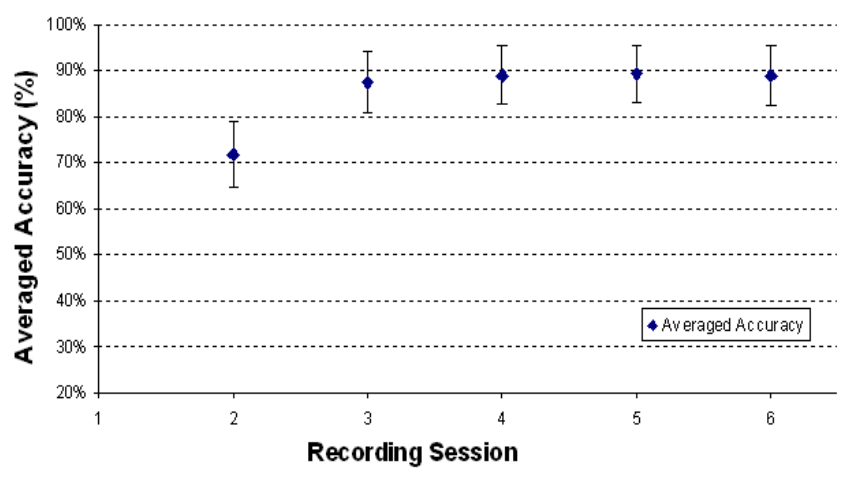

Fig. 5 The averaged accuracy obtained for the classifiers trained during different recording sessions. After the third session the performance doesn't increase significantly.

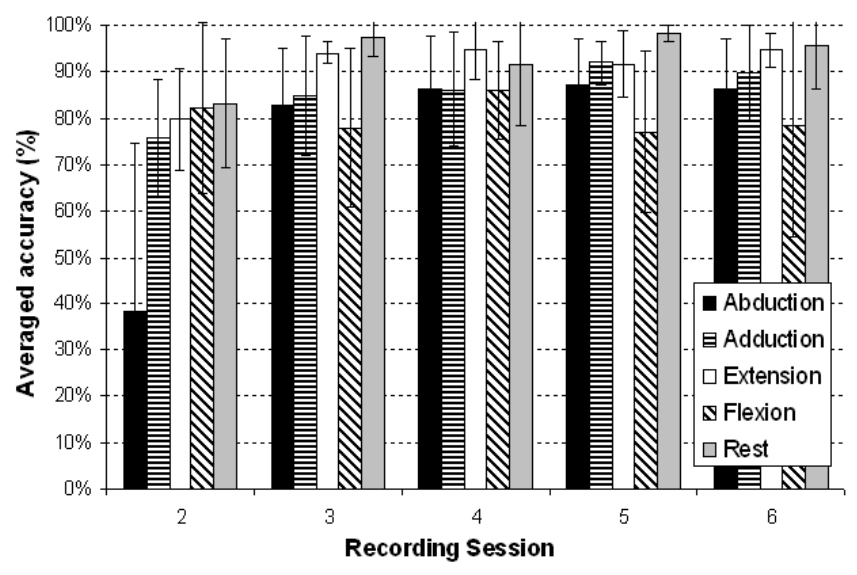

Fig. 6 Progress of the movement classification accuracy over the training sessions. Each class was averaged over all subjects.

Another way to evaluate the classifier performance is shown in Figure 7. Not only are we interested in the correct identification for each movement, we are also concerned with the potential cost associated with misclassification. The sensitivity (or True Positive rate) is plotted against the specificity (or 1 minus the False Positive rate) for each one of the discriminated movements. As this case is not a binary classification problem, the class reference formulation is used [9]. One movement is taken as the positive class and all the others as the negative classes. For every movement, one point in the 'sensitivity-vs-specificity' graph represents the movement performance for a specific recording session. As a result, five points per class are drawn in the graph. They illustrate the behavior of the movement classification through the training 
sessions. The points closer to the upper-right corner represent higher accuracy in the discrimination of movements than points closer to the bottom-left corner. The results illustrated in Figure 7 indicate that the multi-state classifier discriminated both Extension and Rest classes more accurately than Flexion class. Classification of Abduction and Adduction movements presented similar behavior.

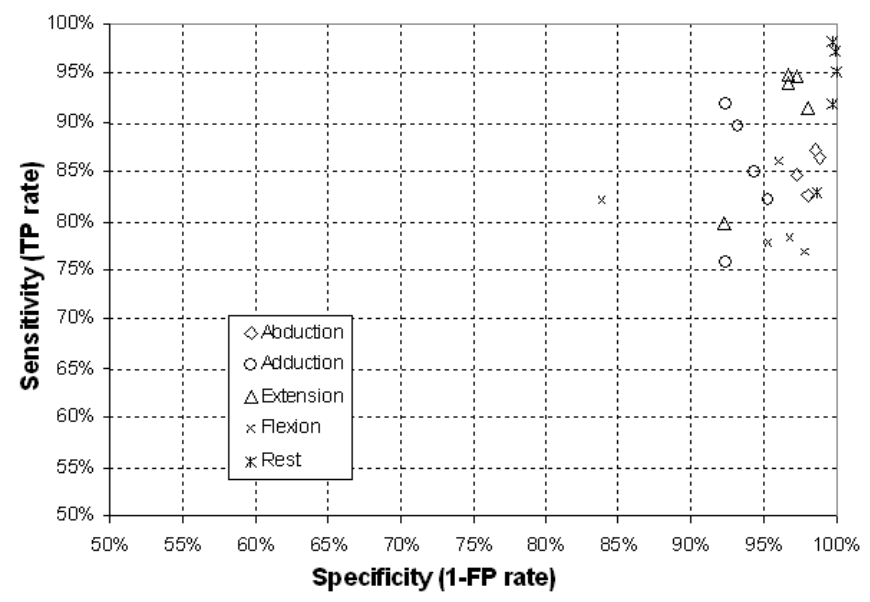

Fig. 7 The sensitivity and specificity for each classified state is illustrated. Each movement contains five points corresponding to the five training sessions.

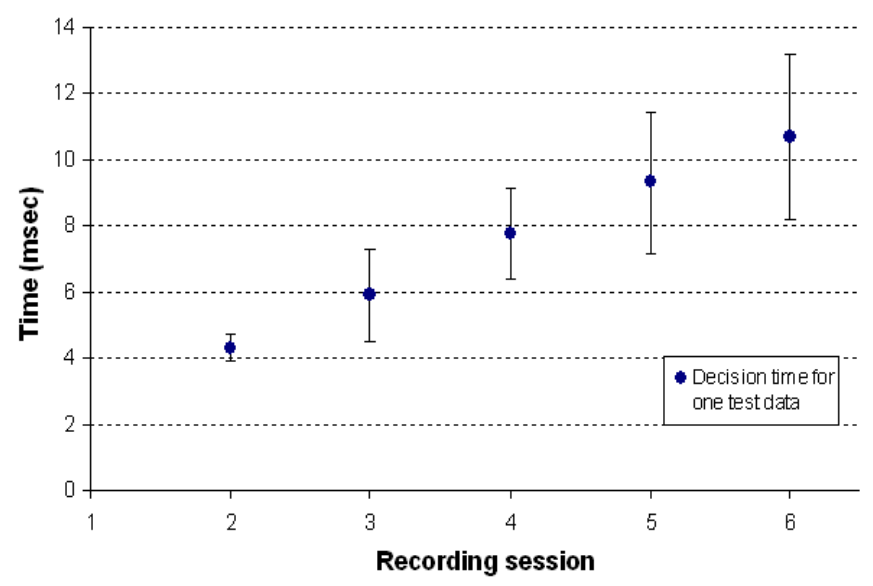

Fig. 8 The progress of the average test time over the recording sessions. The time showed corresponds to the classification of one test point.

Figure 8 shows the average processing time for the classifier to determine the intended movement from the EMG signal in the test set. It increases with the number of recording sessions used in the training state due to an increased number of support vectors used to define the Am. J. Biomed. Sci. 2009, 1(4), 336-343; doi: 10.5099/aj090400336 decision boundary. As a result, the number of matrix multiplications carried out through the decision path also increase, meaning that more time is required to make the final decision.

\section{Discussion}

The performance of the multiclass classifiers is illustrated in Figure 5. It shows an improvement in the classification accuracy that asymptotically approaches $90 \%$. After a critical number of recording sessions, the classifier accuracy starts to reach a plateau, meaning that inclusions of new training data will not significantly improve the performance of the multi-state classifier. This particular study showed that an average of three sessions is needed to achieve classification accuracy higher than $89 \%$. This value may be increased by combining an additional algorithm able to determine the best combination of the averaged wavelet features for this application. Principal component analysis (PCA) algorithm for dimensionality has been proved to work effectively in combination with wavelet transform [10]. The inclusion of PCA in conjunction with SVM is underway.

The Extension and Rest classes can be classified with high accuracy (>90\%) from early sessions, meaning that a small data set is required for their discrimination among the other movements, as illustrated in Figure 7. However, increased amount of data recorded for the Flexion movement did not directly translate to a high Flexion classification accuracy. A potential increase could be gained by increasing the number of repetitions performed during each recording session. As outlined in Figure 7, all movements can be distinguished with high specificity. The specificity-vs-sensitivity data for the Flexion movement is saturated lower than the other classes, meaning that the classifier is able to reject false Flexion points than accepting true Flexion points. Abduction and Adduction movements are classified with good accuracy $(>85 \%)$ when the multiclass classifier is trained with data from more than three recording sessions.

The training strategy proposed attempted to simulate the variations in electrode placement by recording EMG signals during consecutive (C) 2009 by NWPII. All rights reserved. 
sessions. It was based on the fact that small shifts in the electrode position were expected because it is hard to match their exact location in each session. The results obtained for the pattern recognition system implemented support the hypothesis that the training strategy proposed allows the classifier to adapt to the changes produced by alterations in the electrode location. This will permit the amputee to still manipulate its prosthetic device effectively given the fact that either the socket was placed incorrectly on the residual limb or it has been moved from its original location due to its constant use.

When implementing the system in real time, the controller delay is an important parameter. From the onset of the contraction to the class decision, a threshold of $300 \mathrm{~ms}$ is considered acceptable [1]. In this experiment, when training the model with data from three recording sessions, an average of $7.75 \mathrm{~ms}$ is the delay introduced to discriminate one test point from five classes (1.99GHz Pentium 4 based workstation). This is a suitable delay that encourages the future development of a real-time application of the system.

\section{v. Conclusion}

Multiclass Support Vector Machines were trained to discriminate among five classes corresponding to four wrist movements and one resting position. The application of a new training strategy was presented to overcome the problems caused by electrode placement variations. It was shown that at least three sessions are required to discriminate the classes with more than $89 \%$ of accuracy. Depending on the movement, EMG signals corresponding to Flexion is the most difficult for classification. Building on the results from this work, modifications to this pattern recognition system is now underway to incorporate real time aspects of signal classification.

\section{References}

1. Englehart, K.; Hudgins, B.; A robust, real-time control scheme for multifunction myoelectric control, IEEE Transactions on Biomedical Engineering, 2003, 50, 848-854.

2. Hudgins, B.; Parker, P.; Scott, R.N.; A new strategy for multifunction myoelectric control, IEEE Transactions on Biomedical Engineering, 1993, 40, 82-94.

3. Hargrove, L.; Englehart, K.; Hudgins, B.; The effect of electrode displacements on pattern recognition based myoelectric control, EMBS 28th Annual International Conference of the IEEE Engineering in Medicine and Biology Society, 2006, 2203-2206.

4. Englehart, K.; Hudgins, B.; Parker, P.A.; Stevenson, M. Classification of the myoelectric signal using time-frequency based representations, Medical Engineering and Physics, 1999, 21, 431-438.

5. Cortes, C.; Vapnik, V.; Support-vector networks, Ma-chine Learning, 1995, 20, 273 297.

6. Bishop, C. M. Pattern Recognition and Machine Learning. New York, USA, 2006.

7. Platt, J.; Cristianini, N.; Shawe-Taylor, J. Large margin DAGs for multiclass classification, Advances in Neural Information Processing Systems, 2000, 12, 547-553.

8. Hsu, C.W.; Lin, C.J.; A comparison of methods for multiclass support vector machines, IEEE Transactions on Neural Networks, 2002, 13, 415-425.

9. Fawcett, T.; An introduction to ROC analysis, Pattern Recognition Letters, 2006, 27, 861874.

10. Englehart, K.; Hudgins, B.; Parker, P.A. A wavelet-based continuous classification scheme for multifunc-tion myoelectric control, IEEE Transactions on Bio-medical Engineering, 2001, 48, 302-311. 